\title{
NEW SUBFAMILY OF MEROMORPHIC CONVEX FUNCTIONS IN CIRCULAR DOMAIN INVOLVING $q$-OPERATOR
}

\author{
BAKHTIAR AHMAD* AND MUHAMMAD ARIF \\ Department of Mathematics, Abdul Wali Khan University Mardan, KP, Pakistan \\ *Corresponding author: pirbakhtiarbacha@gmail.com
}

\begin{abstract}
The main object of the present paper is to investigate a number of useful properties such as sufficiency criteria, distortion bounds, coefficient estimates, radius of starlikness and radius of convexity for a new subclass of meromorphic convex functions, which are defined here by means of a newly defined $q$-linear differential operator.
\end{abstract}

\section{Introduction AND Definitions}

Quantum calculus ( $q$-calculus), which is the study of classical calculus without the notion of limits, attracted the researchers because of its applications in various branches of mathematics, physics and various other branches of science, for details see $[6,7]$.The $q$-analogue of derivative and integral operators were introduced by Jackson [13,14] along with some applications of $q$-calculus. Later on Aral and Gupta [5-7] introduced the $q$-Baskakov Durrmeyer operator by using $q$-beta function while the author's in $[4,8,9]$ discussed the $q$ generalization of complex operators known as $q$-Picard and $q$-Gauss-Weierstrass singular integral operators. Kanas and Răducanu [15] gave the $q$-analogue of Ruscheweyh differential operator using the concepts of convolution and then studied some of its properties. More applications of this operator can be seen in the paper [3].

Received $13^{\text {th }}$ September, 2017; accepted $4^{\text {th }}$ December, 2017; published $3^{\text {rd }}$ January, 2018.

2010 Mathematics Subject Classification. 30C45, 30C50.

Key words and phrases. Meromorphic functions; Janowski functions; $q$-differential operator.

(C) 2018 Authors retain the copyrights of their papers, and all open access articles are distributed under the terms of the Creative Commons Attribution License. 
In this paper a $q$-differential operator for meromorphic functions using convolution is defined. We use this operator to define and study some properties of a family of meromorphic convex functions associated with circular domain.

Let $\mathfrak{A}$ denote the family of all meromorphic functions $f$ that are analytic in the punctured disc $\mathbb{D}=$ $\{z \in \mathbb{C}: 0<|z|<1\}$ and satisfying the normalization

$$
f(z)=\frac{1}{z^{p}}+\sum_{k=1}^{\infty} a_{k+p} z^{k+p}, \quad(z \in \mathbb{D}) .
$$

Also let $\mathcal{M S}^{*}(\alpha)$ and $\mathcal{M K}(\alpha)$ denote the well known families of meromorphic starlike and meromorphic convex functions of order $\alpha(0 \leq \alpha<1)$ respectively.

For $f$ and $g$ be two meromorphic functions that are analytic in $\mathbb{D}$ and have the form (1.1), then convolution of these functions can be defined by

$$
f(z) * g(z)=\frac{1}{z^{p}}+\sum_{k=1}^{\infty} a_{k+p} b_{k+p} z^{k+p}, \quad(z \in \mathbb{D}) .
$$

For $0<q<1$, the $q$-derivative of a function $f$ is defined by

$$
\partial_{q} f(z)=\frac{f(q z)-f(z)}{z(q-1)}, \quad(z \neq 0, q \neq 1) .
$$

Simple calculations yields that for $n \in \mathbb{N}:=\{1,2,3, \ldots\}$ and $z \in \mathbb{D}$

$$
\partial_{q}\left\{\sum_{n=1}^{\infty} a_{n} z^{n}\right\}=\sum_{n=1}^{\infty}[n, q] a_{n} z^{n-1},
$$

where

$$
[n, q]=\frac{1-q^{n}}{1-q}=1+\sum_{l=1}^{n} q^{l}, \quad[0, q]=0 .
$$

For any non-negative integer $n$ the $q$-number shift factorial is defined by

$$
[n, q] !=\left\{\begin{array}{l}
1, n=0 \\
{[1, q][2, q][3, q] \cdots[n, q], n \in \mathbb{N}}
\end{array}\right.
$$

Also the $q$-generalized Pochhammer symbol for $x \in \mathbb{R}$ is given by

$$
[x, q]_{n}=\left\{\begin{array}{l}
1, n=0, \\
{[x, q][x+1, q] \ldots[x+n-1, q], n \in \mathbb{N}}
\end{array}\right.
$$

and for $x>0$, let $q$-gamma function is defined as

$$
\Gamma_{q}(x+1)=[x, q] \Gamma_{q}(t) \text { and } \Gamma_{q}(1)=1
$$

We now define a function

$$
\Phi_{p}(q, \mu ; z)=\frac{1}{z^{p}}+\sum_{n=1}^{\infty} \Lambda_{n+p} z^{n+p},(\mu>-1, z \in \mathbb{D})
$$


with

$$
\Lambda_{n+p}=\frac{\Gamma_{q}(\mu+n+p+1)}{\Gamma_{q}(\mu+1)[n+p, q] !}=\frac{[\mu+1, q]_{n+p}}{[n+p, q] !} .
$$

It is quite clear that the series defined in (1.4) is convergent absolutely in $\mathbb{D}$. Using the function $\Phi(q, \mu ; z)$ and definition of $q$-derivative along with the idea of convolutions, we now define the differential operator $\mathcal{L}_{q}^{\mu+p-1}: \mathfrak{A}_{p} \rightarrow \mathfrak{A}_{p}$ by

$$
\mathcal{L}_{q}^{\mu+p-1} f(z)=\Phi_{p}(q, \mu ; z) * f(z)=\frac{1}{z^{p}}+\sum_{n=1}^{\infty} \Lambda_{n+p} a_{n} z^{n+p}, \quad(\mu>-1, z \in \mathbb{D})
$$

Also for more details on the $q$-analogue of differential operators see the work $[1,2,17]$.

Motivated from the work studied in $[10,12,18-20]$, we now define a subfamily $\mathcal{M C}_{q}^{*}(p, \mu, A, B)$ of $\mathfrak{A}_{p}$ by using the operator $\mathcal{L}_{q}^{\mu}$ as follows;

Definition 1.1. Let $-1 \leq B<A \leq 1$ and $0<q<1$. Then a function $f \in \mathfrak{A}_{p}$ is in the class $\mathcal{M C}_{q}^{*}(p, \mu, A, B)$, if it satisfies

$$
\frac{-q^{p} \partial_{q}\left(z \partial_{q} \mathcal{L}_{q}^{\mu+p-1} f(z)\right)}{[p, q] \partial_{q} \mathcal{L}_{q}^{\mu+p-1} f(z)} \prec \frac{1+A z}{1+B z}
$$

where the notation " $\prec$ " denotes the familiar subordinations.

Equivalently, a function $f \in \mathfrak{A}_{p}$ is in the class $\mathcal{M C}_{q}^{*}(p, \mu, A, B)$, if and only if

$$
\left|\frac{q^{p} \partial_{q}\left(z \partial_{q} \mathcal{L}_{q}^{\mu+p-1} f(z)\right)+[p, q] \partial_{q} \mathcal{L}_{q}^{\mu+p-1} f(z)}{A[p, q] \partial_{q} \mathcal{L}_{q}^{\mu+p-1} f(z)+B q^{p} \partial_{q}\left(z \partial_{q} \mathcal{L}_{q}^{\mu+p-1} f(z)\right)}\right|<1
$$

\section{The Main Results and Their Consequences}

Theorem 2.1. Let $f \in \mathfrak{A}_{p}$ be of the form (1.1) and satisfy the inequality

$$
\sum_{n=1}^{\infty} \frac{q^{p}[n+p, q][\mu+1, q]_{n+p}}{[n+p, q] !}\left(q^{p}[n+p, q](1+B)+(1+A)[p, q]\right)\left|a_{n+p}\right| \leq[p, q]^{2}(A-B) .
$$

Then the function $f \in \mathcal{M C}_{q}^{*}(p, \mu, A, B)$.

Proof. To show $f \in \mathcal{M C}_{q}^{*}(p, \mu, A, B)$, we only need to prove the inequality (1.7). For this using (1.5), and then with the help of (1.2) and (1.3) we have

$$
\left|\frac{q^{p} \partial_{q}\left(z \partial_{q} \mathcal{L}_{q}^{\mu+p-1} f(z)\right)+[p, q] \partial_{q} \mathcal{L}_{q}^{\mu+p-1} f(z)}{A[p, q] \partial_{q} \mathcal{L}_{q}^{\mu+p-1} f(z)+B q^{p} \partial_{q}\left(z \partial_{q} \mathcal{L}_{q}^{\mu+p-1} f(z)\right)}\right|
$$




$$
\begin{aligned}
& =\left|\frac{q^{p}\left(\frac{[p, q]^{2}}{q^{2 p} z^{p+1}}+\sum_{n=1}^{\infty} \Lambda_{n+p}[n+p, q]^{2} a_{n+p} z^{n+p-1}\right)+[p, q]\left(-\frac{[p, q]}{q^{p} z^{p+1}}+\sum_{n=1}^{\infty} \Lambda_{n+p}[n+p, q] a_{n+p} z^{n+p-1}\right)}{A[p, q]\left(-\frac{[p, q]}{q^{p} z^{p+1}}+\sum_{n=1}^{\infty} \Lambda_{n+p}[n+p, q] a_{n+p} z^{n+p-1}\right)+B q^{p}\left(\frac{[p, q]^{2}}{q^{2 p} z^{p+1}}+\sum_{n=1}^{\infty} \Lambda_{n+p}[n+p, q]^{2} a_{n+p} z^{n+p-1}\right)}\right| \\
& =\left|\frac{\sum_{n=1}^{\infty} \Lambda_{n+p}[n+p, q]\left(q^{p}[n+p, q]+[p, q]\right) a_{n+p} z^{n+p-1}}{-\frac{(A-B)[p, q]^{2}}{q^{p} z p+1}+\sum_{n=1}^{\infty} \Lambda_{n+p}[n+p, q]\left(A[p, q]+B q^{p}[n+p, q]\right) a_{n+p} z^{n+p-1}}\right| \\
& =\left|\frac{\sum_{n=1}^{\infty} q^{p} \Lambda_{n+p}[n+p, q]\left(q^{p}[n+p, q]+[p, q]\right) a_{n+p} z^{n+2 p}}{(A-B)[p, q]^{2}+\sum_{n=1}^{\infty} q^{p} \Lambda_{n+p}[n+p, q]\left(A[p, q]+B q^{p}[n+p, q]\right) a_{n+p} z^{n+2 p}}\right| \\
& \leq \frac{\sum_{n=1}^{\infty} q^{p} \Lambda_{n+p}[n+p, q]\left(q^{p}[n+p, q]+[p, q]\right)\left|a_{n+p}\right|}{(A-B)[p, q]^{2}-\sum_{n=1}^{\infty} q^{p} \Lambda_{n+p}[n+p, q]\left(A[p, q]+B q^{p}[n+p, q]\right)\left|a_{n+p}\right|}<1,
\end{aligned}
$$

where we have used the inequality (2.1) and this completes the proof.

Theorem 2.2. Let $f \in \mathcal{M C}_{q}^{*}(p, \mu, A, B)$ and has the form (1.1). Then for $|z|=r$

$$
\frac{1}{r^{p}}-\tau_{1} r^{p} \leq|f(z)| \leq \frac{1}{r^{p}}+\tau_{1} r^{p}
$$

where

$$
\tau_{1}=\frac{(A-B)[p, q] ![p, q]^{2}}{q^{p}[\mu+1, q]_{p+1}\left((1+A)[p, q]+q^{p}[p+1, q](1+B)\right)}
$$

Proof. Consider

$$
\begin{aligned}
|f(z)| & =\left|\frac{1}{z^{p}}+\sum_{n=1}^{\infty} a_{n+p} z^{n+p}\right| \\
& \leq \frac{1}{\left|z^{p}\right|}+\sum_{n=1}^{\infty}\left|a_{n+p}\right| \quad|z|^{n+p} \\
& =\frac{1}{r^{p}}+\sum_{n=1}^{\infty}\left|a_{n+p}\right| r^{n+p}
\end{aligned}
$$

As $|z|=r<1$ so $r^{n+p}<r^{p}$ and

$$
|f(z)| \leq \frac{1}{r^{p}}+r^{p} \sum_{n=1}^{\infty}\left|a_{n+p}\right|
$$

Similarly

$$
|f(z)| \geq \frac{1}{r^{p}}-r^{p} \sum_{n=1}^{\infty}\left|a_{n+p}\right|
$$

Since (2.1) implies that

$$
\sum_{n=1}^{\infty} \frac{q^{p}[n+p, q][\mu+1, q]_{n+p}}{[n+p, q] !}\left(q^{p}[n+p, q](1+B)+(1+A)[p, q]\right)\left|a_{n+p}\right| \leq[p, q]^{2}(A-B) .
$$

But

$$
\begin{aligned}
q^{p} \frac{[\mu+1, q]_{p+1}}{[p, q] !}\left((1+A)[p, q]+q^{p}[p+1, q](1+B)\right) \sum_{n=1}^{\infty}\left|a_{n+p}\right| \leq \\
\sum_{n=1}^{\infty} q^{p} \frac{[\mu+1, q]_{n+p}}{[n+p, q] !}\left((1+A)[p, q]+q^{p}[n+p, q](1+B)\right)\left|a_{n+p}\right|
\end{aligned}
$$

Hence

$$
q^{p} \frac{[\mu+1, q]_{p+1}}{[p, q] !}\left((1+A)[p, q]+q^{p}[p+1, q](1+B)\right) \sum_{n=1}^{\infty}\left|a_{n+p}\right| \leq[p, q]^{2}(A-B)
$$


which gives

$$
\sum_{n=1}^{\infty}\left|a_{n+p}\right| \leq \frac{[p, q]^{2}(A-B)[p, q] !}{q^{p}\left((1+A)[p, q]+q^{p}[p+1, q](1+B)\right)[\mu+1, q]_{p+1}}
$$

Now by putting this value in (2.2) and (2.3) we get the required result.

Theorem 2.3. Let $f \in \mathcal{M C}_{q}^{*}(p, \mu, A, B)$ and has the form (1.1). Then for $|z|=r$

$$
\frac{[p, q]_{m}}{q^{m p+\zeta} r^{m+p}}-\tau_{2} r^{p} \leq\left|\partial_{q}^{m} f(z)\right| \leq \frac{[p, q]_{m}}{q^{m p+\zeta} r^{m+p}}+\tau_{2} r^{p}
$$

where

$$
\tau_{2}=\frac{[p, q]^{2}(A-B)[p, q] !}{\left((1+A)[p, q]+q^{p}[p+1, q](1+B)\right)} \text { and } \zeta=\sum_{n=1}^{m} n .
$$

Proof. By the virtue of (1.2) and (1.3), we can write

$$
\partial_{q}^{m} f(z)=\frac{(-1)^{m}[p, q]_{m}}{q^{m p+\zeta} z^{p+m}}+\sum_{n=1}^{\infty}[n+p-(m-1), q]_{m+1} a_{p+n} z^{p+n-m} .
$$

Since $|z|=r<1$ so $r^{p+n-m} \leq r^{p}$ for $m \leq n$ hence

$$
\left|\partial_{q}^{m} f(z)\right| \leq \frac{[p, q]_{m}}{q^{m p+\zeta} r^{m+p}}+r^{p} \sum_{n=1}^{\infty}[n+p-(m-1), q]_{m+1}\left|a_{p+n}\right|,
$$

and similarly

$$
\left|\partial_{q}^{m} f(z)\right| \geq \frac{[p, q]_{m}}{q^{m p+\zeta} r^{m+p}}-r^{p} \sum_{n=1}^{\infty}[n+p-(m-1), q]_{m+1}\left|a_{p+n}\right| .
$$

Now by using (2.1) and the following inequality

$$
\begin{gathered}
q^{p} \frac{\left((1+A)[p, q]+q^{p}[p+1, q](1+B)\right)}{[p, q] !} \sum_{n=1}^{\infty}[\mu+p, q]_{p+n}\left|a_{p+n}\right| \leq \\
\sum_{n=1}^{\infty} q^{p} \frac{[\mu+p, q]_{n+p}}{[n+p, q] !}\left((1+A)[p, q]+q^{p}[p+n, q](1+B)\right)\left|a_{n}\right|,
\end{gathered}
$$

we have

$$
\sum_{n=1}^{\infty}[\mu+p, q]_{n+p}\left|a_{n+p}\right| \leq \frac{(A-B)[p, q]^{2}[p, q] !}{q^{p}\left((1+A)[p, q]+q^{p}[p+1, q](1+B)\right)}
$$

but certainly

$$
\sum_{n=1}^{\infty}[n+p-(m-1), q]_{m+1}\left|a_{p+n}\right| \leq \sum_{n=1}^{\infty}[\mu+p, q]_{n+p}\left|a_{n+p}\right|
$$

which implies

$$
\sum_{n=1}^{\infty}[n+p-(m-1), q]_{m+1}\left|a_{p+n}\right| \leq \frac{(A-B)[p, q]^{2}[p, q] !}{q^{p}\left((1+A)[p, q]+q^{p}[p+1, q](1+B)\right)} .
$$

Finally, using this in (2.4) and (2.5) we obtain the required result.

Theorem 2.4. Let $f \in \mathcal{M C}_{q}^{*}(p, \mu, A, B)$. Then $f \in \mathcal{M C}_{p}(\alpha)$ for $|z|<r_{1}$, where

$$
r_{1}=\left(\frac{p(p-\alpha) q^{p}\left((1+A)[p, q]+q^{p}[p+n, q](1+B)\right)[\mu+p, q]_{n+p}}{(p+n)(p+n+\alpha)(A-B)[p, q]^{2}[n+p-1, q] !}\right)^{\frac{1}{n+2 p}} .
$$


Proof. Let $f \in \mathcal{M C}_{q}^{*}(p, \mu, A, B)$. To prove $f \in \mathcal{M C}_{p}(\alpha)$, we only need to show

$$
\left|\frac{z f^{\prime \prime}(z)+(p+1) f^{\prime}(z)}{z f^{\prime \prime}(z)+(1+2 \alpha-p) f^{\prime}(z)}\right| \leq 1
$$

Using (1.1) along with some simple computation yields

$$
\sum_{n=1}^{\infty} \frac{(p+n)(n+p+\alpha)}{p(p-\alpha)}\left|a_{n+p}\right||z|^{n+2 p} \leq 1
$$

From (2.1), we can easily obtain that

$$
\sum_{n=1}^{\infty} \frac{q^{p}[p+n, q][\mu+p, q]_{n+p}}{[n+p, q] !}\left(\frac{\left((1+A)[p, q]+q^{p}[p+n, q](1+B)\right)}{(A-B)[p, q]^{2}}\right)\left|a_{n+p}\right|<1 .
$$

Now inequality (2.6) will be true, if the following holds

$$
\sum_{n=1}^{\infty} \frac{(p+n)(n+p+\alpha)}{p(p-\alpha)}\left|a_{n+p}\right||z|^{n+2 p}<\sum_{n=1}^{\infty} \frac{q^{p}[\mu+p, q] n+p}{[n+p-1, q] !}\left(\frac{\left((1+A)[p, q]+q^{p}[p+n, q](1+B)\right)}{(A-B)[p, q]^{2}}\right)\left|a_{n+p}\right|,
$$

which implies that

$$
|z|^{n+2 p}<\frac{p(p-\alpha) q^{p}\left((1+A)[p, q]+q^{p}[p+n, q](1+B)\right)[\mu+p, q]_{n+p}}{(p+n)(p+n+\alpha)(A-B)[p, q]^{2}[n+p-1, q] !},
$$

and so

$$
\begin{aligned}
|z| & <\left(\frac{p(p-\alpha) q^{p}\left((1+A)[p, q]+q^{p}[p+n, q](1+B)\right)[\mu+p, q]_{n+p}}{(p+n)(p+n+\alpha)(A-B)[p, q]^{2}[n+p-1, q] !}\right)^{\frac{1}{n+2 p}} \\
& =r_{1}
\end{aligned}
$$

we get the required condition.

Theorem 2.5. Let $f \in \mathcal{M C}_{q}^{*}(p, \mu, A, B)$. Then $f \in \mathcal{M S}_{p}^{*}(\alpha)$ for $|z|<r_{2}$, where

$$
r_{2}=\left(\frac{(p-\alpha) q^{p}\left((1+A)[p, q]+q^{p}[p+n, q](1+B)\right)[\mu+p, q]_{n+p}}{(p+n+\alpha)(A-B)[p, q]^{2}[n+p-1, q] !}\right)^{\frac{1}{n+2 p}} .
$$

Proof. We know that $f \in \mathcal{M S}_{p}^{*}(\alpha)$, if and only if

$$
\left|\frac{z f^{\prime}(z)+p f(z)}{z f^{\prime}(z)-(p-2 \alpha) f(z)}\right| \leq 1
$$

Using (1.1) and upon simplification yields

$$
\sum_{n=1}^{\infty}\left(\frac{n+p+\alpha}{p-\alpha}\right)\left|a_{n+p}\right||z|^{n+2 p} \leq 1
$$

Now from (2.1) we can easily obtain

$$
\sum_{n=1}^{\infty} \frac{q^{p}[\mu+p, q]_{n+p}}{[n+p-1, q] !}\left(\frac{\left((1+A)[p, q]+q^{p}[p+n, q](1+B)\right)}{(A-B)[p, q]^{2}}\right)\left|a_{n+p}\right|<1 .
$$

For inequality (2.7) to be true it will be enough if

$$
\sum_{n=1}^{\infty}\left(\frac{n+p+\alpha}{p-\alpha}\right)\left|a_{n+p}\right||z|^{n+2 p}<\sum_{n=1}^{\infty} \frac{q^{p}[\mu+p, q]_{n+p}}{[n+p-1, q] !}\left(\frac{\left((1+A)[p, q]+q^{p}[p+n, q](1+B)\right)}{(A-B)[p, q]^{2}}\right)\left|a_{n+p}\right| .
$$


This gives

$$
|z|^{n+2 p}<\frac{(p-\alpha) q^{p}\left((1+A)[p, q]+q^{p}[p+n, q](1+B)\right)[\mu+p, q]_{n+p}}{(p+n+\alpha)(A-B)[p, q]^{2}[n+p-1, q] !}
$$

and hence

$$
|z|<\left(\frac{(p-\alpha) q^{p}\left((1+A)[p, q]+q^{p}[p+n, q](1+B)\right)[\mu+p, q]_{n+p}}{(p+n+\alpha)(A-B)[p, q]^{2}[n+p-1, q] !}\right)^{\frac{1}{n+2 p}}=r_{2} .
$$

Thus we obtain the required result.

\section{REFERENCES}

[1] I. Aldawish and M. Darus, Starlikness of $q$-differential operator involving quantum calculus, Korean J. Math., 22(4)(2014), $699-709$.

[2] H. Aldweby and M. Darus, A subclass of harmonic univalent functions associated with $q$-analogue of Dziok-Srivastava operator, ISRN Math. Anal., 2013(2013), Article ID 382312, 6 pages.

[3] H. Aldweby and M. Darus, Some subordination results on $q$-analogue of Ruscheweyh differential operator, Abstr. Appl. Anal., 2014(2014), Article ID 958563, 6 pages.

[4] A. Aral, On the generalized Picard and Gauss Weierstrass singular integrals, J. Compu. Anal. Appl., 8(3)(2006), 249 - 261.

[5] A. Aral and V. Gupta, Generalized q-Baskakov operators, Math. Slovaca, 61(4)(2011), $619-634$.

[6] A. Aral and V. Gupta, On the Durrmeyer type modification of the $q$-Baskakov type operators, Non-linear Anal. Theory, Methods and Appl., 72(3 - 4)(2010), 1171 - 1180.

[7] A. Aral and V. Gupta, On q-Baskakov type operators, Demonstratio Mathematica, 42(1)(2009), 109 - 122.

[8] G. A. Anastassiu and S. G. Gal, Geometric and approximation properties of generalized singular integrals, J. Korean Math. Soci., 23(2)(2006), $425-443$.

[9] G. A. Anastassiu and S. G. Gal, Geometric and approximation properties of some singular integrals in the unit disk, J. Inequ. Appl., 2006(2016), Article ID 17231, 19 pages.

[10] J. Dziok, G. Murugusundaramoorthy and J. Sokol, On certain class of meromorphic functions with positive coefcients, Acta Mathematica Scientia B, 32(4)(2012), 1 - 16.

[11] M. R. Ganigi and B. A. Uralegaddi, New criteria for meromorphic univalent functions, Bull. Math. Soc. Sci. Math. Roumanie (N.S.), 33(81)(1989), $9-13$.

[12] A. Huda and M. Darus, Integral operator defined by $q$-analogue of Liu-Srivastava operator, Studia Univ. Babes-Bolyai Ser. Math. 58(4)(2013), $529-537$.

[13] F. H. Jackson, On q-definite integrals, The Quarterly J. Pure Appl. Math., 41(1910), 193 - 203.

[14] F. H. Jackson, On $q$-functions and a certain difference operator, Trans. Royal Soc. Edinburgh, 46(2)(1909), 253 - 281.

[15] S. Kanas and D. Răducanu, Some class of analytic functions related to conic domains, Math. Slovaca 64(5)(2014), 1183 1196.

[16] M. S. Liu, On a subclass of $p$-valent close to convex functions of type $\alpha$ and order $\beta$, J. Math. Study, 30 (1) (1997), $102-104$ (Chinese)

[17] A. Mohammed and M. Darus, A generalized operator involving the $q$-hypergeometric function, Mat. Vesnik, 65(4)(2013), $454-465$.

[18] C. Pommerenke, On meromorphic starlike functions, Pac. J. Math. 13(1963), $221-235$. 
[19] T. M. Seoudy and M. K. Aouf, Coefficient estimates of new classes of $q$-starlike and $q$-convex functions of complex order, J. Math. Inequal. 10(1)(2016), $135-145$.

[20] B. A. Uralegaddi and C. Somanatha, Certain diferential operators for meromorphic functions, Houston J. Math. 17(2)(1991), $279-284$. 